\title{
ESTUDIO Y SIMULACIÓN DE UN SISTEMA DE VIGILANCIA AERONÁUTICA PARA EL CONTROL DE APROXIMACIÓN DE AERONAVES A EL AEROPUERTO EL DORADO, BASADO EN LA TECNOLOGÍA MLAT (MULTILATERACIÓN)
}

Pablo German Perez Herrera

Magister en Ciencias de la Información y las Comunicaciones Universidades Manuela Beltran pgphpollo@yahoo.com.mx Bogotá, Colombia

Tipo: Artículo reporte de caso

Fecha de Recepción: Enero 11 de 2013 Fecha de Aceptación: Marzo 9 de 2013

\section{STUDY AND SIMULATION OF AIRCRAFT SURVEIL- LANCE SYSTEM FOR AIRCRAFT APPROACH CONTROL AT THE EL DORADO AIRPORT, BASED ON MLAT (MUL- TILATERALIZATION) TECHNOLOGY}

\begin{abstract}
The special administrative civil aviation unit (FAA) as an air traffic management service provider, is responsible for the planning, investment and operation of installations, navigation and surveillance of air traffic in order to guarantee security in all airspaces of our Nation. Today, the concept of multilateralization begins to have a global significance in air traffic control management, for upper and lower airspace. In our environment, the FAA requires proposals that allow evaluating the viability of this type of model for implementation in the future, to control aircraft approach to the Dorado Airport. This document aims to provide the civil aviation a serious document that identify and describe the technical and operational feasibility of this system. The main conclusion is reached that MLAT is an excellent choice in surveillance aircraft, and its implementation would allow the Colombian government to increase the application of several free trade agreements with the rest of the world.
\end{abstract}

Key words: coverage, multilateration system, surveillance and control, technical process.

\section{RESUMEN}

La unidad administrativa especial de aeronáutica civil (UAEAC) como proveedor de servicios de gestión de tráfico aéreo se encarga de la planificación, inversión y puesta en funcionamiento de instalaciones, navegación y vigilancia de gestión del tráfico aéreo con el fin de garantizar la seguridad en todos los espacios aéreos de la Nación. En la actualidad el concepto de Multilaterización comienza a tener gran importancia en el control de tráfico aéreo a nivel mundial para la administración de espacios aéreos superiores e inferiores. En nuestro medio la UAEAC requiere propuestas que le permitan evaluar la viabilidad de este tipo de modelos para su implementación en un futuro, para el control de aproximación de 
aeronaves a él aeropuerto El Dorado. El presente documento pretende dotar a la aeronáutica civil de un documento serio, que identifique y formule la viabilidad técnica y operativa de este sistema. Como conclusión principal se llega que MLAT es una excelente opción en vigilancia aeronáutica, y su implementación le permitiría al estado colombiano incrementar la aplicación de diversos acuerdos de libre comercio con el resto del mundo.

Palabras claves: cobertura, sistema de multilateralización, proceso técnico, vigilancia y control.

\section{INTRODUCCIÓN}

La Unidad Administrativa Especial de Aeronáutica Civil (UAEAC) como proveedor de servicios de Gestión de Tráfico Aéreo (ATM) [7], tiene responsabilidad en la planificación, inversión y puesta en funcionamiento de instalaciones y servicios CNS/ATM (que es el encargado de la Comunicación, Navegación y Vigilancia de Gestión del Tráfico Aereo), con el fin de garantizar la seguridad en todos los espacios aéreos de la Nación. Para coordinar las operaciones aéreas en aproximación al aeropuerto El Dorado, el terminal aéreo cuenta con un servicio de gestión del tránsito aéreo ATS, capaz de controlar y supervisar las aeronaves tanto en tierra como en vuelo. Este servicio se divide en navegación, comunicación por medio un circuito oral tierra-aire, tierra-tierra y vigilancia por medio de vectores de aproximación, siendo este último el de interés en el presente artículo. La vigilancia utiliza como herramienta principal el Radar [2], infraestructura en tierra que permite a los controladores aéreos visualizar las aeronaves en un indicador panorámico. Para detectar la distancia y posición acimutal de las aeronaves en un rango determinado se emplea el radar primario, y con el fin de obtener un completo reconocimiento de la aeronave, se emplea el sistema de Radar secundario, el cual proporciona información adicional como altura e identificación de la aeronave.

La multilateralización como técnica de vigilancia de aeronaves [1], comienza a tener gran importancia en el ATC (Control de Tráfico Aereo) mundial para el control de los espacios aéreos superiores e inferiores. La UAEAC [3] necesita un estudio investigativo serio que le permita conocer la viabilidad de un proyecto similar y su implementación en un futuro próximo, para el control de aproximación de aeronaves a él aeropuerto El Dorado. El propósito de este proyecto es el de dotar a la Aeronáutica civil de un documento serio, que identifique y formule la viabilidad técnica y operativa de este sistema en la aproximación El Dorado.

\section{ANTECEDENTES}

El CNA (Centro Nacional de Aeronavegación) Bogotá, ejerce el control de aproximación al Aeropuerto Internacional El Dorado, con un sistema Radar PSR/SSR (Primary Radar System/ Secondary Surveillance Radar) de 60MN instalado en el año 1992 pero operativo desde el año 1994. Este sistema ofrece al controlador de tráfico aéreo, información de posición e identificación de una aeronave en control de aproximación, mediante presentación actualizada en pantallas de radar, con ciclos de refresco de sus imágenes cada 4.8 segundos.

A mediados del año 2010 la UAEA, actualizó del Radar SSR, llevándolo a operación en el modo MSSR (Monopulse SSR), evitando congestiones en la pantalla del controlador, debido a la interrogación selectiva de la nave que se está cuestionando. De todas formas, el MSSR mantiene las deficiencias del SSR, relacionadas con su limitado modo de operación por condiciones topográficas y de cobertura radar. 


\section{MARCO TEÓRICO}

\subsection{Radar secundario SSR}

Se define el radar secundario como un sistema en el cual se interroga de acuerdo a un Modo mediante una señal radioeléctrica emitida por la estación en tierra, la cual inicia a bordo de la aeronave la emisión de una respuesta codificada.

En este sistema, el blanco es un elemento activo ya que se genera una conversación entre la estación en tierra y el transponder, equipo a bordo de la aeronave [6].

Al recibir el equipo de a bordo el modo de interrogación, inicia la transmisión de nuevas señales radioeléctricas en contestación a las interrogaciones recibidas. Estas señales se conocen como códigos o claves que son combinaciones de impulsos previamente seleccionados.

Los dos impulsos que definen el modo son P1 y P3 los cuales tiene un ancho de $0,8 \mu \mathrm{s}$. El impulso P2 separado $2 \mu$ s de P1 tiene como misión controlar los lóbulos secundarios del patrón de radiación. Existen seis modos diferentes de los cuales cuatro son los que usa la OACI para su empleo en ATC Civil, estos se detallan en la figura 1.

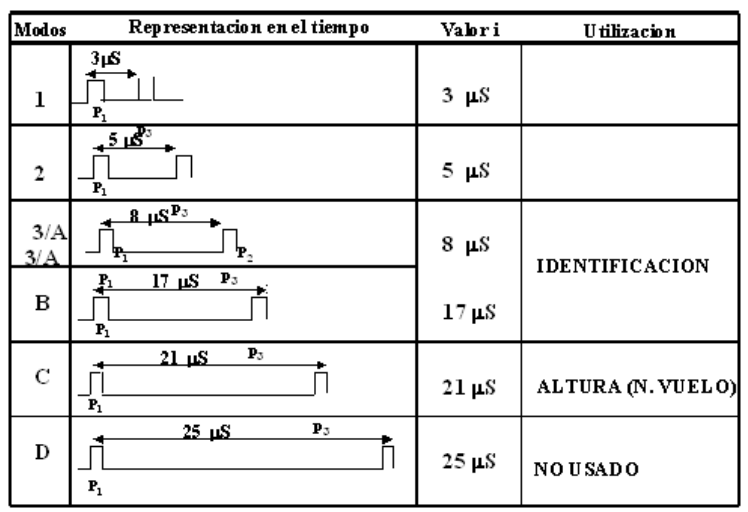

Figura 1. Modos de interrogación.

(Obtenido de Les RadarsDans L' Aviation Civile. ENAC)

Las señales del transponder llamadas claves consisten en un par de impulsos llamado de encuadramiento, separados $20,3 \mu$ s y nombrados como F1 y F2 respectivamente. El ancho de estos impulsos es de $0,45 \mu$ s independientemente del modo empleado para interrogar. Los Transponders de 4096 códigos intercalan 12 impulsos entre los impulsos de encuadramiento F1 y

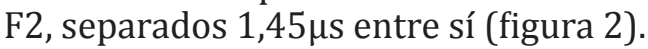

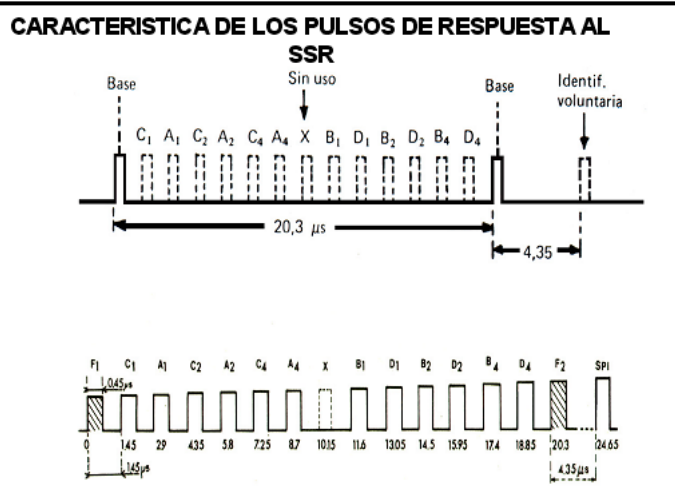

Figura 2. Pulsos definiendo código o claves.

(Obtenido de Les RadarsDans L' AviationCivile. ENAC)

12 pulsos de información binaria 1 y 0 , son intercalados entre F1 y F2, separados $1,45 \mu$ s entre sí. Estos 12 pulsos de información pueden adoptar $212=4096$ valores o códigos distintos, que contienen información de identificación y altitud.

\subsection{Multilateración}

Las técnicas de multilateración se reconocen como un medio viable de vigilancia. En esta técnica de vigilancia 3 ó más estaciones de tierra reciben de forma pasiva señales provenientes de una aeronave y usando una técnica de tiempo de llegada, la posición y la altitud del objetivo pueden ser determinadas. Esta técnica de vigilancia en la actualidad está siendo usada ampliamente y aun cuando sus primeras aplicaciones han sido para la vigilancia en la superficie de los aeropuertos, ya se comienzan a desarrollar aplicaciones para el ATC (control del tráfico aéreo) de aproximación (APP) y de ruta (ACC). 


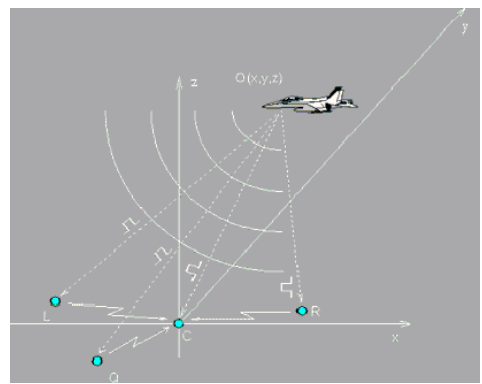

Figura 3. Visualización gráfica de la multilateración.

Es una forma de vigilancia, que consiste en un número de antenas recibiendo una señal desde una aeronave que ha sido interrogada y una unidad de procesamiento central calculando la posición de la aeronave a partir del TDOA de la señal a las diferentes antenas (figura 3).

El TDOA entre dos antenas corresponde matemáticamente hablando con un hiperboloide en 2D sobre el cual se localiza la aeronave. Cuatro antenas detectando la señal desde una aeronave permiten obtener una posición en 3D de la aeronave, al calcular la intersección de los hiperboloides resultantes.

\subsection{Comparación SSR-MLAT}

Se debe hacer énfasis en las mejoras operativas que la multilateración ofrece, sobre la vigilancia Radar. Las principales ventajas son:

\subsubsection{Medición de altura}

El Radar secundario de vigilancia no puede medir altura. El interrogador SSR [5] conoce la altura barométrica de la aeronave, es decir no está en capacidad de, con sus emisiones electromagnéticas, calcular la posición vertical de la aeronave.

Por el contrario, la MLAT si calcula la altura de la aeronave. Por medio de sus estaciones receptoras y de la creación de las hipérbolas, este sistema calcula la posición de la aeronave en tres dimensiones posicionándola correctamente.

\subsubsection{Zonas de vacío}

El Radar se ve afectado por las condiciones del terreno creando zonas o conos de silencio, donde no se tiene información alguna de si una aeronave esta en ese lugar. En otras palabras, cualquier obstáculo que el radar tenga a su alrededor será causante de que no se detecte una aeronave [8].

Por el contrario las estaciones receptoras de MLAT se posicionan para que no existan conos de silencio debido a que están estratégicamente localizadas para cubrir grandes áreas.

\subsubsection{Mejora en pistas paralelas}

Mientras que la separación horizontal entre aeronaves usando el radar es de 5NM, en la multilateración se puede realizar de 3NM [9], esto en pistas paralelas ó casi paralelas garantizaría resultados favorables, aumentando el rendimiento del aeropuerto significativamente con respecto a las operaciones diarias. Caso del aeropuerto El Dorado.

\subsubsection{Refrescos más rápidos}

El tiempo de renovación de la señal del SSR es de aproximadamente 4,8s (tiempo de rotación de la antena), no es malo, pero se sabe que en aviación un segundo es mucho tiempo dado a las velocidades a las que viajan las aeronaves. Esta es una de las mejoras significativas de la MLAT [5], puesto a que el refresco de sus pantallas es de nada más un segundo.

\section{DESCRIPCIÓN DEL PROYECTO}

La multilateración a la hora de ser implementada, debe contar con una ubicación estratégica de sus estaciones, la cual es propia y característica de cada aplicación que se quiera dar al sistema de Multilateración. Esta ubicación estratégica considera el levantamiento geográfico de cada estación y la determinación de distancias existentes entre cada una de ellas, para generar finalmente un diseño bidimensional llamado BASELINE.

Para la generación del BASELINE se debe realizar el análisis de la influencia de la topografía del área sobre el sistema de Multilateración 
que será implementado en el APP de Bogotá.

Se elaborará el mapa de ubicación más óptimo de las estaciones receptoras MLAT, estaciones centrales de procesamiento de datos y estaciones monitoras y de control, con la ayuda de software de aplicación Radiomobile, Google Earth y mapa departamental de Cundinamarca, IGAC.

A partir del BASELINE, se obtendrá un modelo simulado en MatLab que permita posicionar una aeronave en el espacio aéreo de nivel inferior controlado por el ATC (Air Traffic Control) de El Dorado, utilizando la tecnología MLAT [8] y las señales del radar SSR.

Los elementos que participan en este modelo de investigación son:

- La señal SSR.

- $\quad$ El TOA (Time arrival).

- El TDOA (Time Difference of Arrival).

- La serie de hipérbolas definidas por cada estación MLAT, ubicada en los focos y que proporcionan la estimación de la localización de la aeronave.

Las variables que describen el estado de estos elementos son:

- La distancia entre una aeronave y una estación receptora

- La distancia entre las estaciones receptoras MLAT.

- El tiempo necesario para que la señal de la aeronave llegue hasta la antena receptora.

- La posición de la aeronave, que debe resolverse para las tres incógnitas $\mathrm{x}, \mathrm{y}, \mathrm{z}$

- Los lugares específicos de las estaciones receptoras, donde, $x_{\dot{p}} y_{\dot{p}^{\prime}} z_{i^{\prime}} ; x_{j}, y_{j^{\prime}} z_{j^{\prime}} ; x_{k^{\prime}} y_{k^{\prime}} z_{k^{\prime}}$ son de la posición i-enésima, j-enésima y kenésima de las antenas receptoras, respectivamente, y estas posiciones varían con el tiempo [10].

\subsection{Procedimientos APP}

En el aeropuerto internacional El Dorado de la ciudad de Bogotá [13], se realizan aproximacio- nes por instrumentos APP (Approach Control), al ser autorizado únicamente transito IFR (Instrument Flight Rules).

La trayectoria de aproximación está dividida en tres sectores (figura 4).

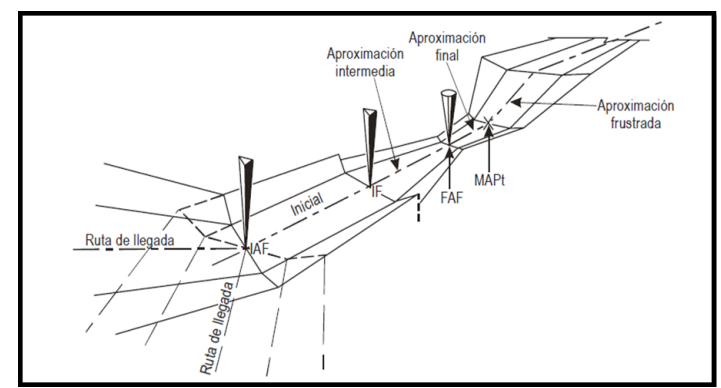

Figura 4. Trayectoria de aproximación.

(Obtenido de Procedimientos, operación de aeronaves volumen I p. I-4-1-10).

Aproximación inicial IAF (initial approach): Parte de un procedimiento de aproximación por instrumentos que se inicia en el punto o referencia de aproximación inicial determinado.

Aproximación intermedia IF (intermediate approach): Parte de un procedimiento de aproximación por instrumentos que se inicia en el punto o referencia de aproximación intermedia determinado.

Aproximación final FAP (final approach): Parte de un procedimiento de aproximación por instrumentos que se inicia en el punto ó referencia de aproximación final determinado [12].

\subsection{Estudio topográfico}

El propósito del estudio topográfico es el de definir el área de aproximación al aeropuerto El Dorado de Bogotá con su respectivo espacio aéreo, determinando su extensión y sus límites, con el fin de proveer en ésta el cubrimiento completo por parte del sistema de multilateración propuesto. Esta área dependerá del espacio definido para aproximación hacia el aeropuerto El Dorado.

En la figura 5, se puede observar en línea verde y dentro de la región central del TMA de Bogo-

Estudio y simulación de un sistema de vigilancia aeronáutica para el control de aproximación de aeronaves a el aeropuerto el dorado, basado en la tecnología MLAT (multilateración) 
tá, el área de aproximación al aeropuerto internacional El Dorado, demarcado por el VOR de la estación IAF de Ambalema, el punto de notificación obligatorio IAF de Utica [11], el VORDME del IF Bogotá y el punto de notificación obligatorio FAP.

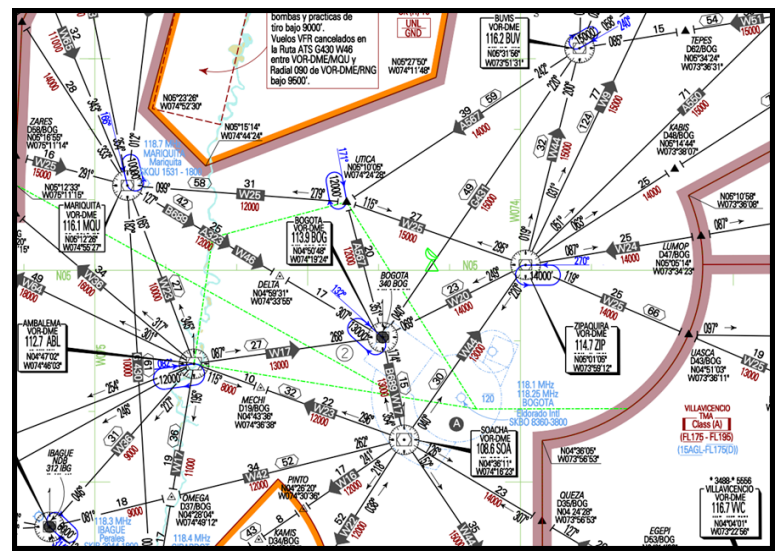

Figura 5. Sector centro TMA Bogotá.

(Obtenido de porción del TMA Bogotá. AIP Colombia, AD 2 SKBO 11. Aeronáutica civil de Colombia).

La figura 6, nos muestra la carta de aproximación por instrumentos hacia el aeropuerto El Dorado.

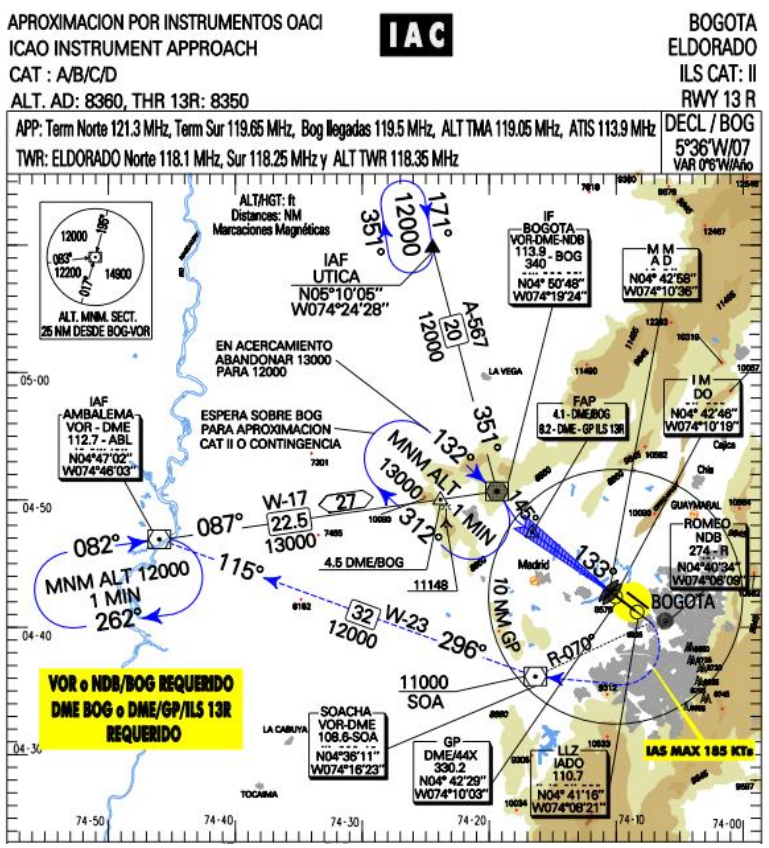

Figura 6. Carta aproximación por instrumentos, El Dorado Bogotá.
Análisis topográfico: La zona centro del TMA BOG, es una región que cubre aproximadamente $1800 \mathrm{Km} 2$ de superficie en el departamento de Cundinamarca y pequeña parte del Departamento del Tolima, en la cual se pueden encontrar alturas en el terreno desde los $300 \mathrm{~m}$ hasta los 3800m (figura 7).

Esto permite identificar la dificultad con la que se debe contar al instalar el sistema de multilateración, el cual se basa en comunicaciones en línea vista [11], pues la característica propia del terreno tan dispar obliga a localizar estaciones en lugares con alturas considerables, lo que permite ampliar el rango de alcance de la señal para cada estación, proporcionando un cubrimiento mayor.

En la figura 8, se puede apreciar lo anteriormente descrito con mayor precisión, La zona centro del TMA BOG con sus correspondientes elevaciones. Esto es de gran utilidad, porque permite identificar los lugares con elevaciones de terreno adecuados para implantar las estaciones MLAT.

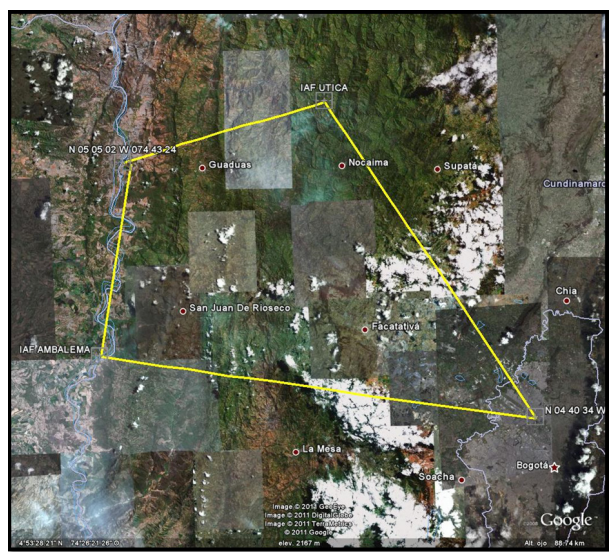

Figura 7. Representación sector centro TMA BOG. Imágenes satelitales (obtenida de Google Earth).

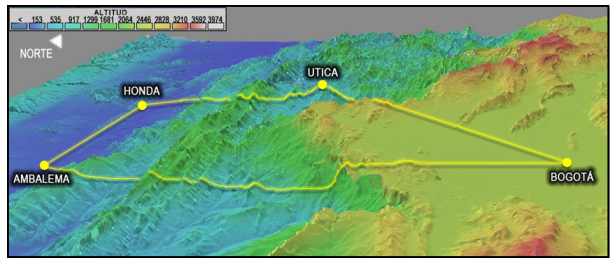

Figura 8. Perspectiva topográfica 3D del sector centro TMA BOG. (Obtenida de Radiomobile). 


\subsection{Determinación del BASELINE}

Se determina la elección de las estaciones MLAT bajo los siguientes criterios:

- Sitios con infraestructura propia y desarrollada, preferiblemente de propiedad de la aeronáutica civil.

- Sitios preferiblemente protegidos contra hechos de orden público.

- Los enlaces de comunicaciones establecidas entre cada una de las estaciones receptoras y la unidad de procesamiento central Bogotá, son vía microondas, estableciendo como prioridad la ubicación de las estaciones para que exista linea de vista LOS (line of sight) [10].

- Diagramas de penetración a 500ft, que ase- guren una cobertura BASELINE de 100\% para la aproximación a El Dorado.

\subsection{Calculo de los enlaces}

Debemos determinar el tipo de antenas que permitan la calidad de la transmisión digital de los datos TOA desde cada una de las estaciones MLAT hacia el centro de control y procesamiento El Dorado (CNA), para asegurar la correlación, el TDOA y el Tracking.

Se da como ejemplo el enlace Andes El Dorado (CNA), figura 9.

La ecuación (1) determinar la ganancia de las antenas.

$$
G(d B i)=\frac{1}{2}\left[\left(\frac{E_{b}}{N_{0}}\right)(d B)-228,6+L_{p}(d B)+R b(d B H z)+10 \log T^{\prime}-P_{T}(d B w)\right]
$$

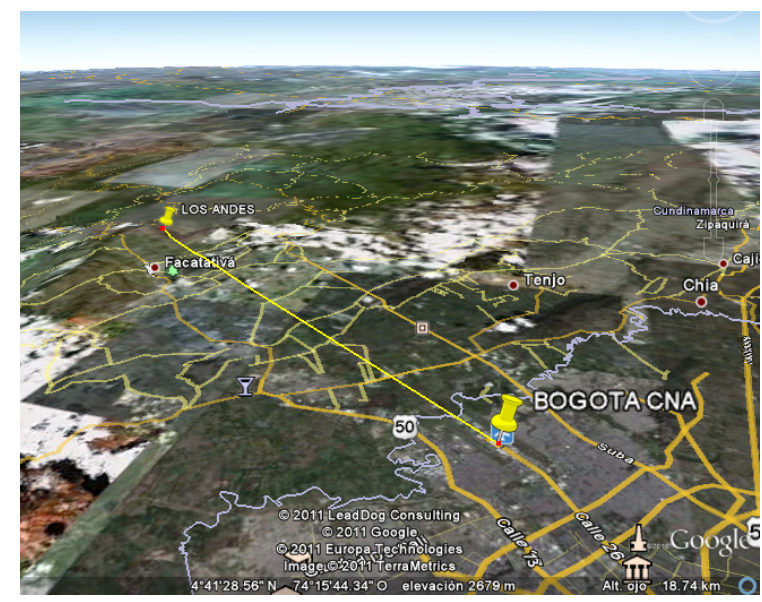

Figura 9. Enlace de microondas estación Los Andes - El Dorado. (Obtenido de GoogleEarth).

\subsection{Simulación MLAT}

Para este simulación se utiliza el método de "multilateración geométrica" basada en la medición del tiempo de llegada de la señal emitida por la aeronave a cada una de las estaciones MLAT, TDO.

Empleando la técnica de posicionamiento por diferencia de tiempos de llegada (TDOA) de estas señales a cada una de las estaciones, se llega al desarrollo del sistema conocido como multilateración, que emplea 4 o más estaciones en fijas en tierra.

La estación central de procesamiento se encarga de procesar los datos y tomar la diferencia de tiempos convirtiéndola en diferencia de distancias para determinar la posición con respecto a las estaciones en tierra de la aeronave.

Para este caso se emplearon seis estaciones en tierra, lo cual determina que las relaciones entre la ubicación de cada una de ellas y la distancia a la aeronave está dada por la ecuación (2), (3), (4), (5), (6), (7).

$$
\begin{aligned}
& R_{1}{ }^{2}=\left(x-x_{1}\right)^{2}+\left(y-y_{1}\right)^{2}+\left(z-z_{1}\right)^{2} \\
& R_{2}^{2}=\left(x-x_{2}\right)^{2}+\left(y-y_{2}\right)^{2}+\left(z-z_{2}\right)^{2} \\
& R_{3}^{2}=\left(x-x_{3}\right)^{2}+\left(y-y_{3}\right)^{2}+\left(z-z_{3}\right)^{2} \\
& R_{4}^{2}=\left(x-x_{4}\right)^{2}+\left(y-y_{4}\right)^{2}+\left(z-z_{4}\right)^{2} \\
& R_{5}^{2}=\left(x-x_{5}\right)^{2}+\left(y-y_{5}\right)^{2}+\left(z-z_{5}\right)^{2} \\
& R_{6}^{2}=\left(x-x_{6}\right)^{2}+\left(y-y_{6}\right)^{2}+\left(z-z_{6}\right)^{2}
\end{aligned}
$$

En donde $R_{n}$ representa la distancia entre cada una de las estaciones hacia la aeronave, $x_{n}$ representan las coordenadas de posicionamiento de las estaciones terrenas en un plano dado y $x$, $y, z$ son las coordenadas de posición de la aero- 
nave respecto al mismo plano.

Teniendo en cuenta los criterios del numeral 4.3 , se eligieron las seis estaciones mostradas en la tabla 2.
La figura 10 muestra el BASELINE obtenido para el APP Bogotá.

La figura 11, muestra la localización de las estaciones RU seleccionadas.

Tabla 2. Estaciones MLAT seleccionadas.

\begin{tabular}{|c|c|c|c|c|c|c|}
\hline Estación & Nombre & Altura (m) & Altura (ft) & Latitud (N) $^{\circ}$ & ${\text { Longitud }(\mathrm{W})^{\circ}}$ & Enlace de datos \\
\hline RU & Cerro Manjuí & 3075,8 & 10091,2 & 4,8385 & 74,4214 & Microondas \\
\hline RU & Los Andes & 3081 & 10108,3 & 4,8752 & 74,3891 & Microondas \\
\hline RU & Mancilla & 3145 & 10318,2 & 4,8725 & 74,3185 & Microondas \\
\hline CPS & Bogotá CNA & 2550 & 8366,1 & 4,6959 & 74,1395 & \\
\hline RU & Pasquilla & 3600 & 11811,0 & 4,4548 & 74,1866 & Microondas \\
\hline RU & Tablazo & 3250 & 10662,7 & 5,0132 & 74,2024 & Microondas \\
\hline RU & Bojacá & 2787 & 9143,7 & 4,7018 & 74,3570 & Microondas \\
\hline
\end{tabular}

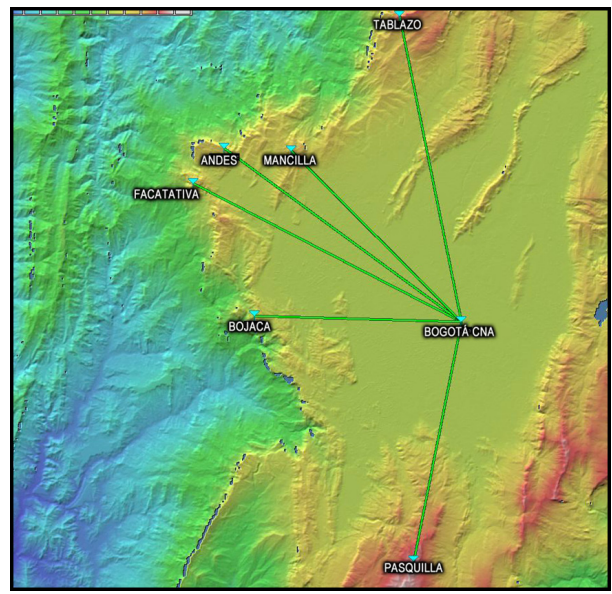

Figura 10. Estaciones MLAT. Enlace de datos.

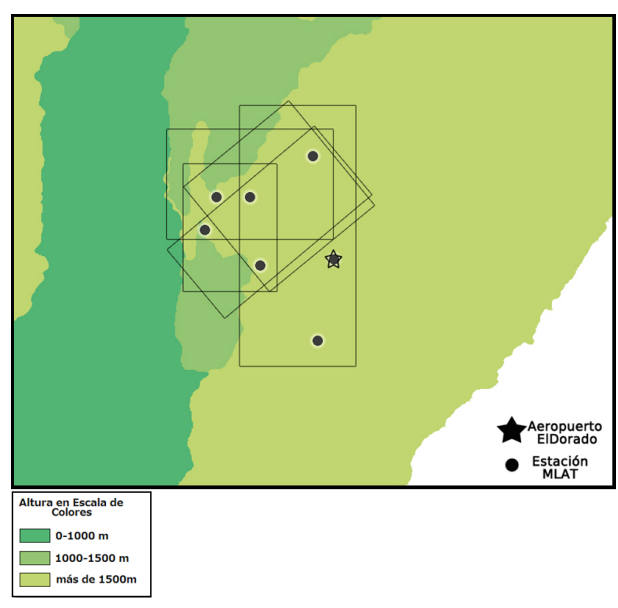

Figura 11. Estaciones RU.
La figura 12, visualiza el cubrimiento proporcionado por las 6 estaciones MLAT a $5000 \mathrm{ft}$ A.M.S.L (altura media sobre el nivel del mar). El resultado es mejor que el esperado, proporcionando cubrimiento a la totalidad del sector centro del TMA BOG.

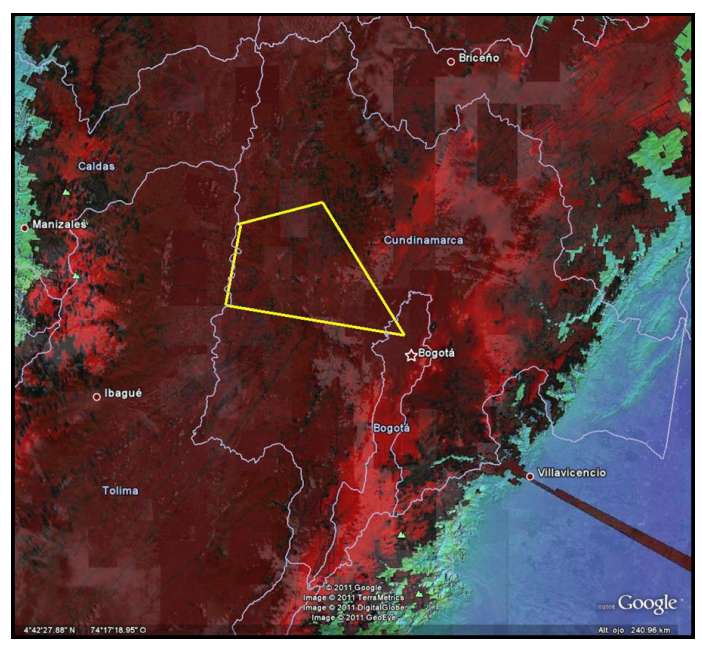

Figura 12. Cubrimiento TMA Bogotá.

\section{SIMULACIÓN MLAT}

El análisis de resultados se realiza en función del caso de estudio, el cual corresponde a un punto denominado Ambalema1. Este representa la ubicación de una aeronave en aproximación al aeropuerto El Dorado, ingresando por 
la ruta de ambalema (figura 13), mediante un procedimiento establecido por la división de aeronavegación de la UAEAC.

El resultado final de cómputo realizado por al algoritmo en MatLab, será una pantalla con una gráfica ubicando la aeronave mediante la interceptación de las seis hipérbolas [4] (figura 14).

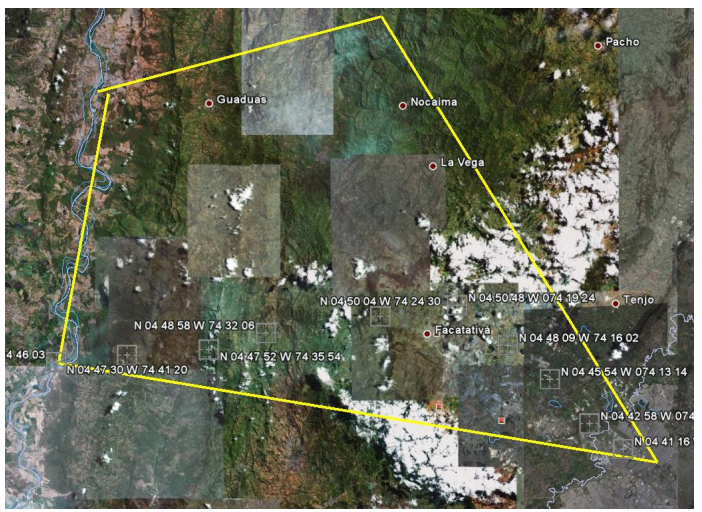

Figura 13. Trayectoria de aproximación por instrumentos: IAF ambalema.

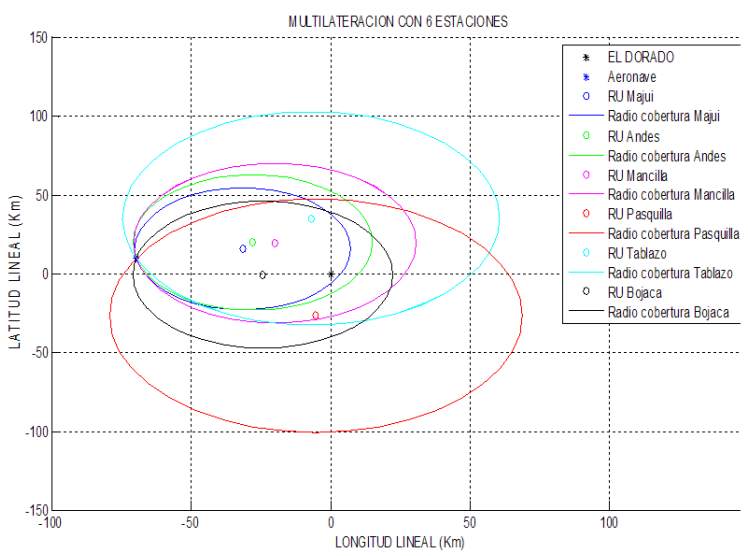

Figura 14. Posición aeronave sobre ambalema 1.

La referencia del sistema (el punto central de coordenadas 0,0 ) resaltado con un asterisco de color negro, es el aeropuerto El Dorado de la ciudad de Bogotá, en torno al cual se aprecian círculos pequeños de diferentes colores, que representan la ubicación de las estaciones que conforman el BASELINE, y cuyas coordenadas geográficas han sido convertidas a coordenadas lineales para facilitar los cálculos del algoritmo, pero que son correspondientes con la ubicación geográfica en longitud y latitud. Se observan además las coberturas hiperboloides de la señal asociada a cada estación.

Haciendo uso del aplicativo desarrollado, la intersección de las coberturas mostraran la posición de la aeronave a partir de los TOA (Time of Arrival) de la señal de $1090 \mathrm{Mhz}$ emitida por el transponder a cada una de las estaciones en tierra.

Simultáneamente, Matlab desplegará una pantalla en la cual se mostrarán los datos de posicionamiento geográfico de la aeronave, calculados por el algoritmo para la multilateración con seis antenas (figura 15).

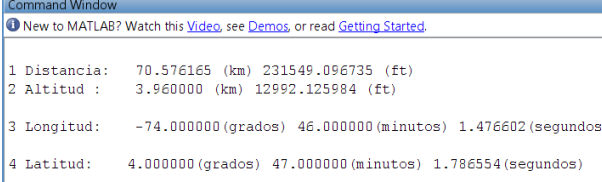

Figura 15. Datos calculados por el algoritmo.

\section{CONCLUSIONES}

La evaluación del estudio de pre-factibilidad técnica se basó en el estudio de las condiciones actuales del aeropuerto, indicando que el aeropuerto en este momento puede operar con sus sistemas actuales de vigilancia, radares ubicados en el Tablazo y en El Dorado (CNA), pero aún con la actual modernización de sus instalaciones y equipos (como el nuevo radar modo S), este cuenta con limitantes, para poder determinar la ubicación real de la aeronave. Con la implementación de otros sistemas de vanguardia tecnológica como la multilateración, se pueden disminuir los problemas presentados actualmente, aumentando los niveles de integridad, confiabilidad, disponibilidad y precisión de los sistemas electrónicos que prestan los servicios de vigilancia en el aeropuerto.

El Approach Bogotá se encuentra en un área bastante accidentada en una perspectiva topográfica, encontrando alturas de hasta $5000 \mathrm{~m}$ como en las cordilleras oriental y central, y planicies de $400 \mathrm{~m}$ sobre el nivel del mar, lo que dificulta la propagación de las señales en alta

Estudio y simulación de un sistema de vigilancia aeronáutica para el control de aproximación de aeronaves a el aeropuerto el dorado, basado en la tecnología MLAT (multilateración) 
frecuencia usadas en vigilancia aeronáutica por parte del radar. La multilateración, con su red de estaciones, permite que sean localizadas estaciones en puntos estratégicos, disminuyendo de manera notoria los sectores con debilidad de señal o ausencia total (puntos ciegos).

Las operaciones aéreas aumentaron desde el 2004 y gracias a las nuevas alianzas internacionales (Taca-Avianca-Aeroga;2010,LanChileAires-Aeroasis;2010) que permitirán cubrimiento de más rutas con mayor frecuencia de

\section{Referencias Bibliográficas}

[1] W. Neven, T. Quilter, R. Hogendoom; Wide area multilateration, Versión 1.1, Eurocontrol, August 2005.

[2] Aerocivil; Políticas planes y proyectos institucionales delaUnidadAdministrativa Especializada de Aeronáutica Civil Colombiana. (Bogotá Mayo 2010). [En línea], consultado en Agosto 15 de 2011, disponible en: http://portal.aerocivil.gov. co/portal/page/portal/Aerocivil_Portal_ Internet/Inicio.

[3] D. Muñoz, F. Bouchereau, C. Vargas, R. Enríquez; Position location techniques and application, Burlinghton, EE.UU. 2009.

[4] R. Bucher, R. Misra; three-dimentional hiperbolic Positioning system, New Jersey Institute of Tecnology, 2001.

[5] Eurocontrol; Comparative assessment of SSR vs. Wide Area Multi-Lateration, Edition 1.3. 2005.

[6] B. Sklar, B; Digital communications fundamentals and applications, Editorial Prentice Hall, 1988.

[7] OACI. Doc. 4444 ATM/501; las aerolíneas nacionales e internacionales, las operaciones desde y hacia el aeropuerto $\mathrm{El}$ Dorado van a seguir en aumento, indicando el requerimiento inmediato de sistemas que puedan ayudar a controlar y monitorear el espacio aéreo colombiano controlando y monitoreando el Approach BOG de manera completa en una forma más eficiente. La multilateración es la primera opción en vigilancia aeronáutica, proporcionando un paso intermedio hacia la evolución de la gestión del tráfico aéreo.

[8] AIP Colombia AD 2 - SKBO 1; Aeronáutica Civil de Colombia, 2007

[9] Reglamentos Aeronáuticos de Colombia; Parte sexta, Gestión de Tránsito Aéreo, 2002.

[10] Operación de Aeronaves. Doc. 8168 OPS/611; procedimientos para los servicios de navegación aérea, Edición 5, Volumen I, 2006.

[11] OACI. DOC. 9643 AN/941; Manual sobre operaciones simultaneasen pistas de vuelo por instrumentos paralelas o casi paralelas, Edición 1, 2004.

[12] Laboratorio Nacional Aeroespacial NLR (2005), la NLR-CR-2004-472; Multilateración de área amplia, Informe sobre TRS EATMP 131/04, versión 1.1, 2004.

[13] Instituto Geográfico Agustín Codazzi; Mapa Departamental de Cundinamarca MDI, Topográficos, Escala 1:300000, Bogotá Colombia, 2009. 